Museum (Natural History), is assisting in botanical and photographic work. Other personnel have been drawn from Malaya, Sarawak and North Borneo, with the intention of developing regional interest in the project. The Government of Singapore has made available from the staff of the Singapore Botanic Gardens the services of the botanist, Dr. Chew Wee Lek, and of two plant collectors, Md. Shah and Kadim b. Tassim. Much scientific work on the flora of Borneo has been done at these Gardens, the herbarium of which contains a very valuable set of Bornean plants. Md. Shah is the son of the late Haji Md. Nur, who contributed so much to the scientific work of the Gardens. General vertebrate zoology is in the charge of Prof. J. L. Harrison (Nanyang University), who joined the expedition for a month during June 17-July 22, and Mr. Chin Phui Kong (assistant fisheries officer, North Borneo). Mr. B. E. Smythies (conservator of forests, Sarawak), the author of the recently published book Birds of Borneo, is joining the expedition as ornithologist in July. The Forest Department, North Borneo, is assisting in opening up the intended trail up the eastern shoulder of the mountain and across the so-called Pinosuk Plateau, which is of great scenic and biological interest, but apparently of little use for economic development. Various members of the Department are visiting the expedition in its different stages.
The members of the expedition passed through Singapore to Jesselton at the end of May and are spending from June until mid-September on the slopes of the mountain. It is intended to use a portable aluminium sheet hut as a base camp at an altitude of $5,000 \mathrm{ft}$., whence local expeditions will be made with tents up the unexplored eastern approaches of the mountain. Since exact localization will often be necessary, a set of aerial photographs is being used in conjunction with the War Office and Air Ministry survey maps (1:50,000).

Among the many objects of the expedition, special attention is being given to the soil conditions in the mossy forest at 5,000-7,000 ft. altitude and in the areas of ultrabasic rock, to the occurrence of the great parasitic flower Rafflesia, to the extraordinary variety of wild figs, and to the opportunity for obtaining photographs to illustrate Mr. Corner's durian theory of tropical forests; Borneo being the centre of the remarkable genus of durian trees.

Local arrangements are being made through the office of the Resident, West Coast Residency, North Borneo, and Messrs. Harrisons and Crosfield have undertaken to act as the Expedition's agents in North Borneo. The botanical and zoological results will be co-ordinated by the staffs of the Royal Botanic Gardens, Kew, and the British Museum (Natural History) respectively.

\title{
ATOMIC STRUCTURE OF GRAPHITE AND GRAPHITIZATION
}

$\mathrm{A}^{\mathrm{A}}$ $\mathrm{N}$ informal discussion on graphite, under the auspices of the Faraday Society, was held on January 26 in the Chemical Engineering and Chemical Technology Department of the Imperial College of Science and Technology, London. About 120 people were present, including five from France and one or two others from the Continent.

The programme was opened by the chairman, Prof. A. R. Ubbelohde (Imperial College of Science and Technology), who outlined the scope of the papers to be presented and indicated the kind of problems which it was hoped would be clarified by the papers and subsequent discussion. The first paper, by Prof. C. A. Coulson (Mathematical Institute, University of Oxford), was on the electronic band structure of graphite and the results of a calculation of the energy-levels by conventional tight-binding methods. For the case of a single graphite plane, it is found that the $\pi$ electrons have energy-levels which fall into two half-bands, just touching one another at the ends where the density of states is zero. Under the influence of neighbouring layers the two halfbands broaden and overlap, so that the valance band is not quite full and the upper (conduction) band is not quite empty. From this energy-level distribution, both the bond-length and the shape of the X-ray emission band can be calculated and they are in good agreement with experimental results. Other properties, such as the electrical conductivity and the de Haas-Van Alphen effect, depend only on the nature of the top occupied levels, and reasonable agreement with the calculation in found in practice. Finally, Prof. Coulson discussed the electronic behaviour at the edges of a graphite layer and the possible situations when either neighbouring sites or a single separated site are involved. Both adsorption experi- ments and quenching by oxygen are explicable if it is supposed that there are unpaired electrons at a few sites around the edges.

In the following paper, Dr. G. E. Bacon (Atomic Energy Research Establishment, Harwell) reviewed the outcome of the study of graphite by diffraction methods in terms of : (1) the limited crystallographic perfection; (2) the distribution of electrons around the carbon atoms; (3) the texture of artificial graphites; (4) the effect of irradiation by neutrons. The characteristic features of X-ray powder photographs could be explained in detail in terms of random displacements of the carbon planes about the $c$-axis, with a measureable difference in the inter-layer spacing between oriented and mis-oriented planes. The proportion of rhombohedral modification can be varied by mechanical working or by treatment with strong acids or bromine, but the detailed manner in which the transformation takes place is not understood. A combination of neutron diffraction data, careful measurement of X-ray intensities and theoretical calculations has established that the departures from spherical symmetry in the electronic distribution are very small. Irradiation in a nuclear reactor produces significant changes in macroscopic properties, such as elasticity and bulk dimensions, and although these can be explained in general terms as due to interstitial carbon atoms and vacancies, the d'tailed nature of these defects is still uncertain.

Under the title "Defauts Cristallins dans le Graphite", Prof. J. Mering (Institut National de Recherche Chimique Appliquée, Paris) gave a system. atic classification of the various types of defect which can be found in graphite crystals, and discussed their detection and observation and their influence on chemical and physical properties. At the outset 
he emphasized the outstanding difficulties in this kind of work-the non-existence of a perfect graphite erystal as a reference standard, and the impossibility of introducing or removing in any simple way faults of a given type. The main classification was into point, line and planar faults. Point faults are the simplest type, but they are difficult to detect by direct means, although they produce relatively simple effects on physical properties (such as electrical resistivity and the Hall coefficient) and on the chemical properties, in particular the tendency to form lamellar compounds. These point faults can be subdivided in terms of substitution atoms, vacancies and interstitials. Faults associated with dislocations and with surfaces are the least simple to define and interpret, but they are detectable more directly by diffraction methods and optical and electron microscopy. Planar faults were subdivided into 'surfaces of dislocations' (such as occur between the not-quiteparallel domains in so-called single crystals of natural graphite or between hexagonal and rhombohedral regions) and errors in layer stacking (such as the orientation faults, which are particularly noticeable in artificial graphites, or random mistakes in an $A B$ or $A B C$ stacking sequence).

The second session of the meeting dealt with slightly more technological aspects of graphite and was opened with a paper by Mr. P. K. C. Wiggs (Morgan Crucible Co., Ltd., London) on "Elastic Properties of Graphite". Single crystals of graphite have a Young's modulus parallel to the basal planes which is about three times as high as for steel, but parallel to the $c$-axis the modulus is many times smaller. The easiest mode of deformation is, as would be expected, a shear in which neighbouring planes slide over one another. The properties of polycrystalline graphite bear little resemblance to those of the ideal single crystal, and are determined very largely by the disordered material at the junctions between crystallites and by internal voids : such anisotropy as exists is a consequence of the texture which results from the nature of the starting materials and mode of manufacture. As a consequence of this, it is found that all the elastic moduli of polycrystalline graphite are of the same order of magnitude as the low-shear modulus for the sliding of adjacent planes in the single crystal. Only for the more recent developments of pyrolytic graphite and graphite whiskers, where stresses cannot be relieved by sliding, do the high modulus and strength of the single crystal parallel to the planes become apparent. At temperatures greater than $2,000^{\circ} \mathrm{C}$., electric furnace graphite is deformed plastically to a very significant extent, but on heating up to this temperature there is a two-fold increase in the elastic moduli on account of the increasing thermal motion of the carbon atoms, which reduces the ability of one plane to slide over another.

A paper by Dr. E. G. Steward (Northampton College, London) described the crystallographic changes which occur during graphitization. There is still considerable doubt about the structural details of poorly crystallized material, which gives very diffuse X-ray diffraction patterns. Recently, there has been a revival of suggestions of non-planar structures, such as a diamond-type of bonding, particularly from electron diffraction observations. As graphitization proceeds and the crystallites grow, the interpretation is much clearer : a progressive modulation of the two-dimensional bands and a general sharpening of the reflexions demonstrates the approach towards the ideal Bernal structure. The ease of graphitization depends on the structure of the crystal nuclei and whether these are separated by cross-linkages. Initial growth occurs by incorporation of non-organized carbon at the crystallite edges, but further progress depends on the existence of near-parallel orientation between neighbouring crystallites. An important characteristic of the final product may be the size of the quasi-perfect macrounits which are much larger than the regions of higher structural perfection revealed by X-ray diffraction. Recent developments in the work on pyrolytic carbons and the examination of moiré fringe patterns in the electron microscope were also discussed.

The final paper, by Prof. W. F. K. Wynne-Jones (King's College, Newcastle upon Tyne), dealt with physical and chemical studies of the graphitization process.

A number of questions and general discussion followed each of the sessions. One of the principal topics was the lack of understanding of the transformation from ordinary to rhombohedral graphite and the energy relations between the two forms. It was also evident that we still have little precise knowledge of the intergranular material which bonds together the individual crystallites, and which is so important in determining the physical and chemical properties of ordinary non-ideal material.

G. E. BACON

\section{RADIOLOGICAL UNITS AND MEASUREMENTS}

$\mathrm{R}$ ADIATION dosimetry is an immensely complicated subject and many of its problems have been only partially resolved. The difficulties are of a fundamental nature and arise: $(a)$ from the complexity of the interactions of neutrons and electromagnetic radiations with matter; (b) from the still greater complexity of interaction of charged particles, especially electrons, with matter. So long as the understanding of these basic processes remains incomplete, there will be outstanding problems in the dosimetry of ionizing radiations. There is consequently a recurring need-it amounts to a necessityfor authoritative pronouncements on this developing subject to maintain world-wide uniformity in the units, standards and measurements of radiation and radioantivity. The National Bureau of Standards
Handbook $78^{*}$ is intended to satisfy this need. It supersedes the 1956 report of the International Commission on Radiological Units and Measurements, and the chief interest lies in the difference between the two reports. There has been no major revision and the differences consist almost entirely of accretion and expansion; the new handbook is in fact twice as long as the old. The chief aim according to the preface is ". . . to improve the accuracy of the evaluation of absorbed dose in all places of interest in a patient or other objects". To this end, there is a greatly expanded section on clinical and biological applica-

* United States Department of Commerce: National Bureau of Standards. Handbook No. 78: Report of the International Commission on Radiological Units and Measurements (ICRU), ${ }^{1959}$. Pp $\mathrm{x}+90$.
65 cents. 